\title{
The importance of microstructure in determining polaron generation yield in poly(9,9-dioctylfluorene)
}

\author{
Nathan J. Cheetham, Manuel Ortiz, Aleksandr Perevedentsev, \\ Laura-Isabelle Dion-Bertrand, Gregory M. Greetham, \\ Igor V. Sazanovich, Michael Towrie, Anthony W. Parker, \\ Jenny Nelson, Carlos Silva, Donal D. C. Bradley, \\ Sophia C. Hayes and Paul N. Stavrinou
}

\section{Published version information}

Citation: NJ Cheetham et al. "The importance of microstructure in determining polaron generation yield in poly(9,9-dioctylfluorene)." Chemistry of Materials, vol. 31, no. 17 (2019): 6787-6797.

DOI: $\underline{10.1021 / a c s . c h e m m a t e r .9 b 01256}$

This document is the unedited author's version of a Submitted Work that was subsequently accepted for publication in Chemistry of Materials, copyright (C2019 American Chemical Society, after peer review. To access the final edited and published work see DOl above.

Please cite only the published version using the reference above. This is the citation assigned by the publisher at the time of issuing the AAM. Please check the publisher's website for any updates. 


\section{The importance of microstructure in determining polaron}

\section{generation yield in poly(9,9-dioctylfluorene)}

Nathan J. Cheetham, ${ }^{* 1}$ Manuel Ortiz, ${ }^{3}$ Aleksandr Perevedentsev, ${ }^{4}$ Laura-Isabelle Dion-

Bertrand, ${ }^{3}$ Gregory M. Greetham, ${ }^{5}$ Igor V. Sazanovich, ${ }^{5}$ Michael Towrie, ${ }^{5}$ Anthony W.

Parker, ${ }^{5}$ Jenny Nelson, ${ }^{1}$ Carlos Silva, ${ }^{6,7}$ Donal D. C. Bradley, ${ }^{2,9}$ Sophia C. Hayes, ${ }^{* 8}$ Paul N.

Stavrinou $*, 2$

${ }^{1}$ Department of Physics and Centre for Plastic Electronics, Imperial College London, London SW7 2AZ, UK

${ }^{2}$ Department of Engineering Science, University of Oxford, Oxford, OX1 3PJ, UK

${ }^{3}$ Département de Physique and Regroupement Québécois sur les Matériaux de Pointe, Université de Montréal, Montréal, Québec, H3C 3J7, Canada

${ }^{4}$ Department of Materials, ETH Zürich, Vladimir-Prelog-Weg 5, Zürich, 8093, Switzerland

${ }^{5}$ Central Laser Facility, Research Complex at Harwell, Science and Technology Facilities Council, Rutherford Appleton Laboratory, Harwell, OX11 0QX, UK

${ }^{6}$ School of Physics, Georgia Institute of Technology, Atlanta, Georgia 30332, USA

${ }^{7}$ School of Chemistry and Biochemistry, Georgia Institute of Technology, Atlanta, Georgia 30332, USA

${ }^{8}$ Department of Chemistry, University of Cyprus, P.O. Box 20537, Nicosia 1678, Cyprus

${ }^{9}$ Department of Physics and Division of Mathematical, Physical and Life Sciences, University of Oxford, 9 Parks Road, Oxford OX1 3PD, UK 


\section{Abstract}

Understanding the structure-property relationships that govern exciton dissociation into polarons in conjugated polymers is key in developing materials for optoelectronic applications such as light-emitting diodes and solar cells. Here, the polymer poly $(9,9-$ dioctylfluorene) (PFO), which can form a minority population of chain segments in a distinct, lower-energy ' $\beta$-phase' conformation, is studied to examine the influence of conformation and microstructure on polaron generation in neat thin films. Using ultrafast transient absorption spectroscopy to probe PFO thin films with glassy-phase and $\beta$-phase microstructures, and selectively exciting each phase independently, the dynamics of exciton dissociation are resolved. Ultrafast polaron generation is consistently found to be significantly higher and long-lived in thin films containing $\beta$-phase chain segments, with an average polaron yield that increases by over a factor of three to $4.9 \%$ vs $1.4 \%$ in glassyphase films. The higher polaron yield, attributed to an increased exciton dissociation yield at the interface between conformational phases, is most likely due to a combination of the significant energetic differences between glassy-phase and $\beta$-phase segments and disparities in electronic delocalisation and charge carrier mobilities between phases. 


\section{Introduction}

The molecular arrangement, or microstructure, of semiconducting polymer chains in the solid-state fundamentally affects their photophysical properties. Solid-state microstructure, and the resulting energetic landscape, is one of the most important factors controlling exciton dissociation into polarons in conjugated polymers. In order for exciton dissociation into polaron states to occur, large exciton binding energies much greater than thermal energy must be overcome. ${ }^{1,2}$ For the polyfluorene homopolymer poly(9,9-dioctylfluorene), an exciton binding energy, $E_{b}=300 \pm 100 \mathrm{meV}$ (cf. $k_{B} T=25 \mathrm{meV}$ at room temperature) has previously been estimated (for an as-cast sample). ${ }^{2}$ More complex copolymers, containing electron donating and electron accepting moieties (donor-acceptor or D-A), tend to exhibit lower binding energies as Coulombic attraction is reduced with increased spatial separation of electron wavefunctions. ${ }^{3}$ Likewise, in organic solar cells, blends of electron donor and acceptor materials have been used to induce further spatial separation of charged electronic states. ${ }^{4}$ Intriguingly, in relatively simple homopolymers, where there is an absence of any obvious energetic driving force for exciton dissociation, ultrafast photogeneration of polarons has been observed in several polymers. ${ }^{5-11}$ Therefore, questions pertinent to the fundamental understanding of semiconducting polymers include: (i) How are the driving forces for exciton dissociation in conjugated homopolymers governed by microstructure? (ii) How general are these factors within the extensive family of conjugated polymers?

Recent studies investigating the effect of microstructure on polaron photogeneration in regioregular poly(3-hexylthiophene) (P3HT) have shown that charge generation increases with mass-average molar mass $\left(M_{w}\right) .{ }^{12-15}$ This observation was attributed to a change in microstructure from a single-phase system of polycrystalline domains to a two-phase structure comprising crystalline regions separated by disordered (pseudo-amorphous) chains. In the same vein, increased charge generation in regioregular vs regiorandom $\mathrm{P} 3 \mathrm{HT}$ is 
attributed to the change from an amorphous to a semicrystalline microstructure. ${ }^{16}$ Increased charge carrier mobility has also been measured in better-connected two-phase systems of P3HT, ${ }^{17-20}$ further highlighting the profound influence molecular arrangement has on optoelectronic properties. Reports have also highlighted the importance of electronic delocalisation for enhanced polaron generation. ${ }^{21-23}$ The width of the energetic density of states (DOS, also termed energetic disorder), resulting from conformational variation within populations of polymer chains, together with local effects such as proximity to polar groups and surfaces, has been identified as another critical factor controlling charge generation in neat materials. ${ }^{13}$ Recent theoretical simulations and modelling of D-A heterojunctions further suggest that charge separation is facilitated by or as a result of energetic disorder, ${ }^{24}$ with a broader density of states (higher degree of energetic disorder) decreasing the activation energy barrier to charge separation and hence increasing the separation probability. ${ }^{25-27}$ Further insight into the relationship between microstructure and polaron generation can be gained by the study of conjugated polymers where the energetic DOS and microstructure can be systematically controlled. Hence, in this report, a detailed study of polaron generation in poly(9,9-dioctylfluorene) (PFO) is presented. PFO is chosen because chain segments can adopt a well-defined, highly-ordered ' $\beta$-phase' conformation whose formation can be carefully and reproducibly controlled by a variety of thin film processing methods. ${ }^{28-34}$ In recent years, a growing number of PFO derivatives and related polyfluorenes have also shown a similar $\beta$-phase conformation. ${ }^{35-44}$ Polymer chain segments adopting a $\beta$-phase conformation exhibit a planarised interunit torsion angle of $\approx 180^{\circ}$, in comparison to the majority in-plane isotropic 'glassy-phase' segments which adopt a much wider range of more twisted torsion angles of $\approx 120-140^{\circ} .30,45$ While for $\beta$-phase the alternation of side chains leads to formation of a $2{ }_{1}$ helix, the direction of side chain extension does not necessarily alternate for the glassy-phase. The higher 
planarity of the $\beta$-phase conformation leads to more linearly extended and higher polarizability chains, with $\pi$-interactions delocalised over a longer conjugation length. ${ }^{28,30}$ This results in characteristic lower energy $\beta$-phase electronic transitions and optoelectronic properties that are spectrally distinct compared to the more disordered glassy-phase. ${ }^{28,46,47}$ In addition to being highly-ordered, the $\beta$-phase conformation is also very well-defined: electronic transitions are approximately fixed in energy, independent of the method of induction or the amount of $\beta$-phase, ${ }^{31}$ and exhibit narrow linewidths in both absorption and emission spectra, signalling much lower conformational disorder than the glassyphase. $^{45,46,48,49}$

Unlike the complex semi-crystalline microstructures found for P3HT, with crystalline domains of variable size connected to surrounding disordered regions, ordered chain segments of $\beta$-phase in PFO do not form lamellae and appear reasonably well dispersed within the matrix of glassy chains. ${ }^{30,46,49,50}$ Furthermore, the relatively twisted ground state conformation of glassy-phase PFO (which causes the ground state absorption onset to be significantly higher energy than that of the planarised $\beta$-phase), in combination with the welldefined and distinct nature of the $\beta$-phase conformation, mean that formation of $\beta$-phase segments causes abrupt spectral changes. This is in contrast with the more gradual orderdisorder transitions observed for most conjugated polymers such as $\mathrm{P} 3 \mathrm{HT} .{ }^{51}$ This makes the two phases of PFO straightforward to resolve spectrally, and leads to a relatively simple, twocomponent-like system of energetically well-defined $\beta$-phase chain segments embedded within disordered glassy-phase chains of a much broader conformational and energy range. The unique conformation-controlled microstructure of PFO therefore provides a useful testbed for studying the influence of microstructure, complementing the ubiquitous studies of P3HT. 
By carefully controlling the fraction of low-energy, highly-ordered $\beta$-phase segments within otherwise disordered glassy films, the microstructure and the resulting energetic landscape may be systematically varied for a series of PFO thin films. This has recently been used to study the effects of conformation on strong and ultrastrong coupling in metal-polymer-metal microcavities. ${ }^{52}$ Films containing $\beta$-phase segments in addition to the glassy-phase are often referred to simply as $\beta$-phase PFO samples.

Here, ultrafast femtosecond and continuous-wave photoinduced absorption spectroscopy measurements (referred to throughout as transient absorption, TA, and PIA respectively), are used to reveal the formation and dynamics of polaron, triplet and singlet excited state species, and their dependence on: (i) $\beta$-phase fraction; and (ii) selective excitation of either solely $\beta$ phase chain segments, or predominantly glassy-phase chain segments (the first such report in the literature). Previous studies have reported a qualitative increase in polaron generation for PFO containing $\beta$-phase chain segments vs purely glassy-phase films. ${ }^{48,53-55}$ In the present work, the yield of photogenerated polarons is quantified through a rational fitting procedure of ultrafast TA spectra complemented by quasi-steady-state photoinduced absorption measurements obtained from a series of thin film samples containing different fractions of $\beta$ phase. The polaron generation yield is found to increase significantly upon $\beta$-phase formation, independent of which electronic species is initially excited. This suggests that the interfaces between glassy and $\beta$-phase chain segments are responsible for the enhanced polaron generation. 


\section{Experimental}

\section{Materials and sample preparation}

PFO was synthesised by Suzuki coupling and used as received from Cambridge Display Technology $\left(M_{n}=18,000 \mathrm{~g} \mathrm{~mol}^{-1}, M_{w}=48,600 \mathrm{~g} \mathrm{~mol}^{-1}, Ð=2.7\right)$ and Sumitomo Chemical Company Ltd $\left(M_{n}=97,000 \mathrm{~g} \mathrm{~mol}^{-1}, M_{w}=287,000 \mathrm{~g} \mathrm{~mol}^{-1}, Ð=3.0\right)$. Molar mass values were obtained from gel permeation chromatography calibrated with polystyrene standards. Due to the higher chain stiffness of PFO vs polystyrene, GPC has been shown to overestimate average molar mass by a factor of 2.7 relative to absolute values obtained from coupled GPC and light scattering. ${ }^{28}$ Toluene (HPLC grade, $>99.7 \%$, VWR), decahydronaphthalene (“decalin"; reagent grade, a mixture of cis and trans isomers, Sigma-Aldrich) and 1,8diiodooctane (DIO, 98\% with copper as a stabiliser, Sigma-Aldrich) were used as received. PFO $\left(M_{w}=48,600 \mathrm{~g} \mathrm{~mol}^{-1}, Ð=2.7\right)$ thin films for steady-state optical and TA measurements were deposited onto circular sapphire substrates (12 mm diameter, c-axis cut, UQG Optics Ltd). In-plane isotropic, glassy PFO films were prepared by spin-coating PFO solutions in

toluene $\left(7 \mathrm{mg} \mathrm{mL}^{-1}\right)$ at $3000 \mathrm{rpm}$ for $60 \mathrm{~s}$, with both solution and substrates pre-heated to 100 ${ }^{\circ} \mathrm{C}$ for 2 min immediately prior to deposition. PFO films with $7-8 \% \beta$-phase fractions (details of $\beta$-phase estimations are given further on in this section) were prepared by exposing glassy PFO films to saturated toluene vapour at $40{ }^{\circ} \mathrm{C}$ for 24 hours. Films with $23 \% \beta$-phase content were prepared by drop-casting $0.1 \mathrm{wt} . \%$ PFO solutions in decalin followed by slow solvent evaporation. Film thicknesses for the glassy, $8( \pm 2) \% \beta$-phase and $23( \pm 2) \% \beta$ phase samples are $40 \pm 5 \mathrm{~nm}, 45 \pm 5 \mathrm{~nm}$ and $42 \pm 5 \mathrm{~nm}$ respectively, measured using a Bruker Dektak XT profilometer.

PFO $\left(M_{w}=287,000 \mathrm{~g} \mathrm{~mol}^{-1}, \emptyset=3.0\right)$ thin films for PIA measurements were deposited onto square fused silica substrates (12 x $12 \mathrm{~mm}$, Spectrosil 2000, UQG Optics). Glassy films were 
prepared by spin-coating PFO solutions in toluene $\left(13 \mathrm{mg} \mathrm{mL}^{-1}\right)$ at $2300 \mathrm{rpm}$ for $60 \mathrm{~s}$, with both solution and substrates pre-heated to $100{ }^{\circ} \mathrm{C}$ for $2 \mathrm{~min}$ immediately prior to deposition. Films with $22( \pm 3) \% \beta$-phase were spin-cast from solutions in toluene $\left(8 \mathrm{mg} \mathrm{mL}^{-1}\right)$ with 2 wt. $\%$ DIO at $2000 \mathrm{rpm}$ for $60 \mathrm{~s}$ at room temperature.

\section{Steady-state absorption spectroscopy}

UV-Vis absorption spectra were recorded using a dual-beam Shimadzu UV-2600 spectrophotometer equipped with a diffuse reflectivity (integrating sphere) attachment and corrected for reflection to give absorption $(A(\%)=100-T(\%)-R(\%))$.

\section{Ultrafast transient absorption spectroscopy}

\section{Experimental setup}

Ultrafast transient absorption spectroscopy measurements were performed using the ULTRA setup at the STFC Rutherford Appleton Laboratory, described in detail elsewhere. ${ }^{56}$ In brief, an amplified titanium sapphire laser (Thales Optronique) produces $\sim 50 \mathrm{fs}$ pulses at a repetition rate of $10 \mathrm{kHz}$. The laser fundamental output, $800 \mathrm{~nm}$, is then split into two parts. The first part generates UV pump pulses through an optical parametric amplifier (TOPAS OPA) at either 385 or $437 \mathrm{~nm}$. The temporal FWHM of the pump pulse was observed to be $\sim 150 \mathrm{fs}$, giving $\Delta \lambda= \pm 4 \mathrm{~nm}$ (assuming pulses are transform-limited). The second part of the laser output is used to generate a broadband white light pulse, 520-1090 nm, by focusing the fundamental into a $2 \mathrm{~mm}$ calcium fluoride plate. The probe pulse was also split into two parts. The first part was focused to the sample, with the second used as a reference beam to monitor variations in the laser spectrum and intensity. The transmission of probe pulses (sample and reference) were measured using 512-pixel silicon single-diode arrays.

Pump and probe beam diameters were approximately 160 and $80 \mu \mathrm{m}$, respectively and were overlapped spatially on the sample. Measurements were carried out with pump and probe 
beam polarisation set at an angle of $54.7^{\circ}$ to each other. The pump and probe were modulated at 5 and $10 \mathrm{kHz}$ respectively, allowing the difference spectra between 'probe-only' and 'pump + probe' transmission to be obtained in real time. An optical delay line controlled the time delay between pump and probe. Distortions in the white light probe intensity were present due to the probe beam being coincident with the laser fundamental at $\sim 1.55 \mathrm{eV}$. Consequently, this region of the TA spectra was removed for all data sets.

Thin film samples were held in a helium-cooled cryostat regulated with a temperature controller, either at $290 \mathrm{~K}$ or $15 \mathrm{~K}(\Delta T= \pm 2 \mathrm{~K})$. The cryostat was rastered continuously during measurements in both $x$ and $y$ planes to avoid sample degradation. Photoluminescence emission spectra before and after TA measurements showed no observable changes: this is strong evidence for the absence of sample degradation, as PFO is well-known to exhibit redshifted 'green-band' emission from fluorenone defects upon oxidation. ${ }^{57,58}$

\section{Spectral fitting \& yield estimation}

Prior to fitting, all TA data was first chirp corrected. Following this, TA spectra at each time delay were then fit with a linear superposition of spectral profiles: two Gaussian profiles to represent glassy-phase polaron and triplet excited state absorption; two Lorentzian profiles to represent $\beta$-phase polaron and triplet excited state absorptions (a Lorentzian profile was selected on the basis of providing the best fit to PIA spectra); and the excited state singlet absorption was represented by a linearly scaled 'singlet-only' TA spectrum. For a given sample, the 'singlet-only' spectrum was the TA spectrum at the earliest delay time exhibiting a reliable signal and before the resolution of triplet and polaron signals at later times. This delay time was found to coincide with the peak of the integrated TA spectral area, at $\Delta t=$ 200-300 fs. Spectra were fit between 1.38 and $2.17 \mathrm{eV}$, using least squares fitting. Limits for peak positions and widths for the Gaussian and Lorentzian functions were based on results from photoinduced absorption spectroscopy measurements, and were used to constrain the 
fits. Subsequently, the fitted TA spectral components (excited state singlet, polaron and triplet) were integrated between 1.38 and $2.17 \mathrm{eV}$ at each time delay point to yield excited state kinetics.

To estimate the photogenerated yield of polarons and triplets in PFO, the population of excited state species as a function of time was first determined from integrated TA signals using the following equation derived from the Beer-Lambert-Bouguer law, ${ }^{9,59-61}$

$$
N_{E S}(\Delta t)=\frac{2.303 \cdot \Delta A_{E S}(\Delta t)}{\sigma_{E S} \cdot d}
$$

where $N_{E S}\left[\mathrm{~cm}^{-3}\right]$ is the population of a given excited state species, $\triangle A_{E S}$ is the associated change in sample absorbance (a spectrally integrated area in this study rather than absorbance at a single wavelength), $\sigma_{E S}\left[\mathrm{~cm}^{2}\right]$ is the absorption cross-section and $d[\mathrm{~cm}]$ is film thickness.

Experimentally obtaining absorption cross-sections from TA measurements alone is nontrivial, ${ }^{62}$ often requiring photophysical models. For PFO, there are literature values for some excited states species (obtained from various measurement techniques over separate studies), however there is no self-consistent source containing singlet, polaron and triplet cross-section values. Nevertheless, absorption cross-sections could be estimated using the best available values for PFO and similar fluorene-based polymers: glassy hole polaron, $\sigma_{\text {glassy-pol }}=8 \times 10^{-16}$ $\mathrm{cm}^{2}$ and $\beta$-phase hole polaron, $\sigma_{\beta \text {-pol }}=4 \times 10^{-16} \mathrm{~cm}^{2}$ from spectroelectrochemical measurements of PFO; ${ }^{63,64}$ a lower limit for triplets of $\sigma_{\text {triplet }} \geq 2 \times 10^{-16} \mathrm{~cm}^{2}$ taken from photoinduced absorption spectroscopy of $\mathrm{PFO},{ }^{48}$ and excited state singlet and triplet crosssections for the related step-ladder polyfluorene PIFTO $\left(\sigma_{S l \rightarrow S n}=3 \times 10^{-16} \mathrm{~cm}^{2} \text { from TA }\right)^{9}$ and fluorene copolymer F8BT $\left(\sigma_{\text {triplet }}=3.1 \times 10^{-16} \mathrm{~cm}^{2}\right)$ respectively ${ }^{65}$ Based on these literature values, the absorption cross-sections given in Table 1 were used to estimate excited state populations. The uncertainty in the values is estimated to be $\pm 33 \%$, based on the 
variation between similar $\sigma$ values as well as different methods used to extract $\sigma$ values between reports.

Table 1. Estimated absorption cross-sections, $\sigma$, for excited state species of PFO based on literature values.

\begin{tabular}{|c|c|}
\hline Excited state species & $\boldsymbol{\sigma}\left(\mathbf{c m}^{2}\right)$ \\
\hline Singlet, $S_{1}-S_{n}($ Glassy and/or $\beta$-phase $)$ & $3( \pm 1) \times 10^{-16}$ \\
\hline Triplet, $T_{1}-T_{n}($ Glassy and/or $\beta$-phase $)$ & $3( \pm 1) \times 10^{-16}$ \\
\hline Polaron, $P_{1}-P_{n}($ Glassy-phase $)$ & $8( \pm 2.7) \times 10^{-16}$ \\
\hline Polaron, $P_{1}-P_{n}(\beta$-phase $)$ & $4( \pm 1.3) \times 10^{-16}$ \\
\hline
\end{tabular}

From an initial excited state population dominated by singlet states, the yield of polarons and triplets may be found from the ratio of the maximum populations of polarons or triplets to the maximum population of singlets. Estimated uncertainties for the yields were quantified by the propagation of errors, based on the uncertainties quoted in Table 1, as well as estimated percentage uncertainty of $\pm 20 \%$ for the TA spectral areas obtained from fitting, and the variability in film thicknesses of around $\pm 10 \%$. From this, the percentage uncertainty in the final yields is estimated to be is $\pm 60 \%$.

\section{Photoinduced absorption spectroscopy}

Quasi-steady-state PIA measurements were performed with PFO samples mounted in a closed-cycle cryostat cooled to $12 \mathrm{~K}$. A double modulation technique was used and described in detail elsewhere. ${ }^{66}$ Samples were excited with a continuous wave $406 \mathrm{~nm}$ laser modulated at $170 \mathrm{~Hz}$ and probed by a monochromatic $150 \mathrm{~W}$ tungsten-halogen lamp modulated at 139 Hz. The transmission was measured using a Si avalanche photodiode. Two lock-in amplifiers measured both the fractional change in the probe transmission through the sample, $\Delta T$, referenced to the sum frequency of the pump and probe $(309 \mathrm{~Hz})$; and the probe transmission through the sample, $T$, referenced to the probe modulation frequency. The ratio of these two signals is the presented $\Delta T / T$ spectra. The probe passed through a monochromator before the sample and $\Delta T / T$ was measured incrementally in $2 \mathrm{~nm}$ steps between $550 \mathrm{~nm}$ and $1000 \mathrm{~nm}$. 


\section{Results}

Figure 1 shows steady-state absorption spectra from PFO thin films $(d=40-45 \mathrm{~nm})$ with varying fractions of $\beta$-phase. All samples were prepared using previously reported processing techniques, ${ }^{30,33,67}$ as detailed in the experimental section.
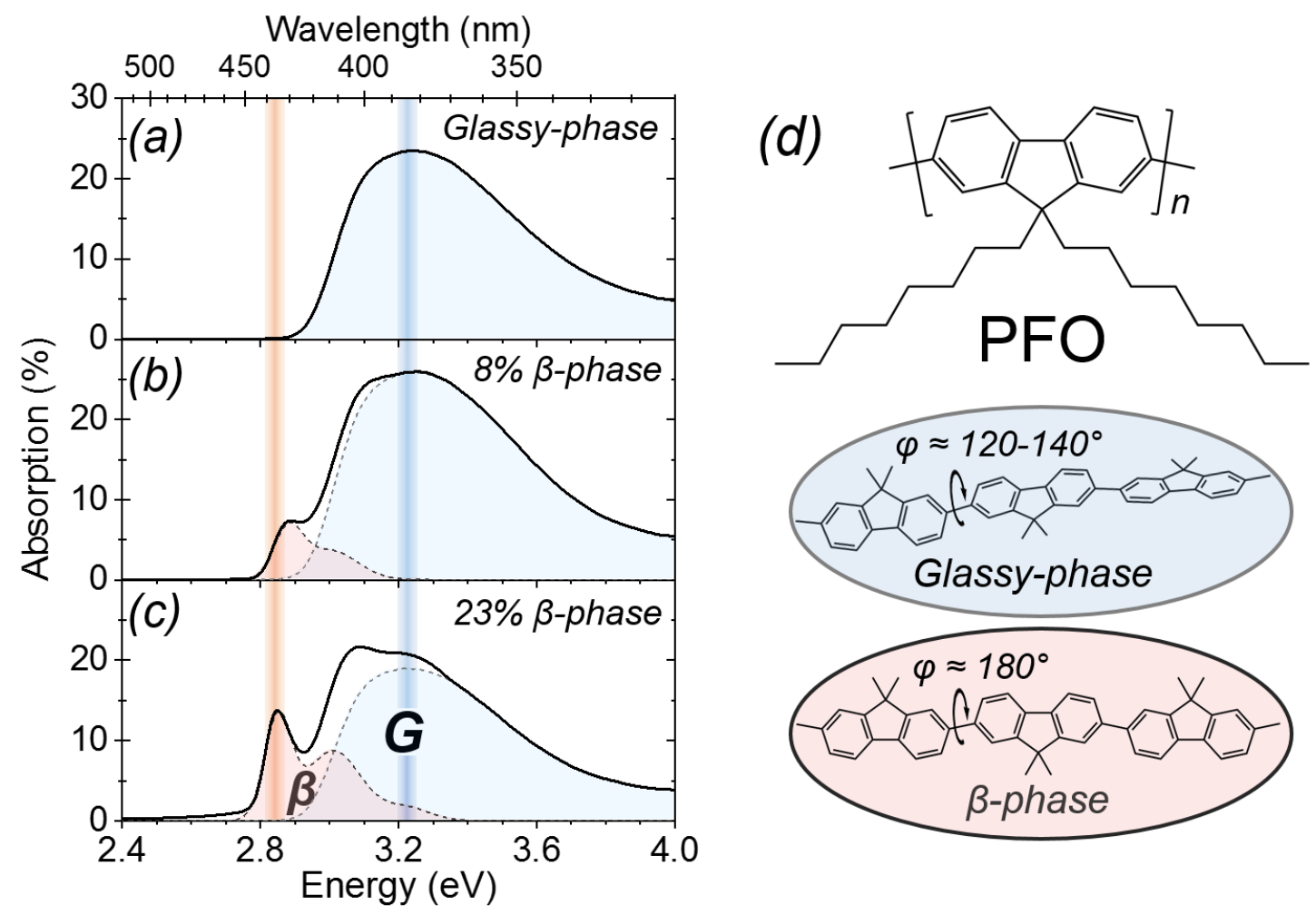

Figure 1. UV-Vis absorption spectra from thin-film samples of PFO comprising: (a) 100\% glassyphase and films with (b) $8( \pm 2) \%$ and (c) $23( \pm 2) \% \beta$-phase chain segments. The UV-Vis spectra are resolved into their glassy-phase ( $G$, blue shading) and $\beta$-phase ( $\beta$, red shading) components. Ultrafast transient absorption spectroscopy excitation wavelengths of $385 \mathrm{~nm}$ (blue band) and $437 \mathrm{~nm}$ (orange band) are highlighted. (d) PFO chemical structure and schematics of chain segments in glassy and $\beta$ conformational phases, with the differences in torsion angle highlighted (alkyl side chains removed for clarity).

The spectra comprise contributions from the absorption profiles of glassy- and $\beta$-phase chain segments. By subtracting the $100 \%$ (pure) glassy-phase spectrum and fitting the resulting difference spectra with a series of Gaussian peaks (see SI Figure S1 and the associated text 
for more details), the glassy and $\beta$-phase contributions can be separated as indicated in Figure 1. Integration of the $\pi-\pi^{*}$ absorption contributions up to $3.88 \mathrm{eV}(320 \mathrm{~nm})$, and correction for the calculated difference in oscillator strength of glassy and $\beta$-phase chain segments, ${ }^{68}$ allows the fraction of $\beta$-phase chain segments to be estimated (using SI equation S1), yielding $8 \pm 2$ $\%$ and $23 \pm 2 \%$. As expected, the formation of $\beta$-phase gives rise to lower energy absorption peaks relative to the disordered glassy-phase, with clearly resolved vibronic structure. ${ }^{30}$ The energy difference between the absorption maxima of the glassy and $\beta$-phase is $380 \mathrm{meV}$. This offset represents a substantial difference in the energetics of the two conformations, which will be present at the interface between glassy and $\beta$-phase segments, both along and between polymer chains. The $\beta$-phase chain segments have also been previously shown to display a notable increase in polarizability (by $80 \%),{ }^{48}$ demonstrating an extended delocalisation of the exciton wavefunction. 
Having established the presence of the $\beta$-phase conformation from steady-state optical spectra, the generation of excited state species is examined. Figure 2 shows ultrafast lowtemperature transient absorption spectra from pure glassy-phase and $23 \% \beta$-phase PFO samples excited at $385 \mathrm{~nm}$ with $\mathrm{FWHM} \approx 150 \mathrm{fs}$ pump pulses.
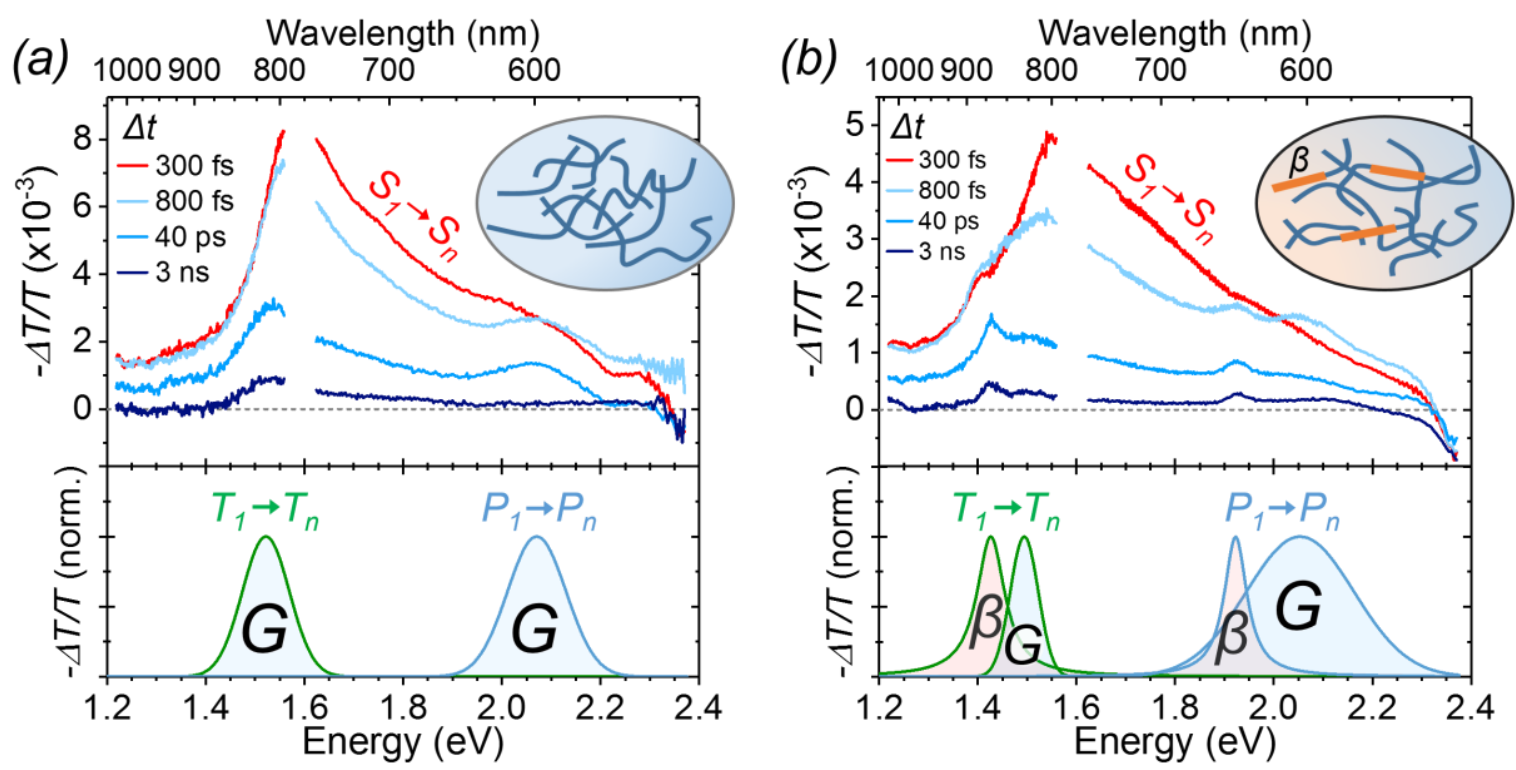

Figure 2. Low temperature transient absorption spectra $\left(T=15 \mathrm{~K}, \lambda_{\text {exc }}=385 \mathrm{~nm}\right.$, pump fluence $=30$

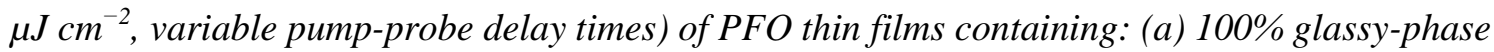
chains and (b) 23 ( \pm 2$) \% \beta$-phase. Early pump-probe delay spectra (red lines) are dominated by the singlet excited state. The lower panels in each sub-plot indicate typical Gaussian and Lorentzian peaks used to represent glassy and $\beta$-phase triplet (shaded green lines) and polaron (shaded blue lines) transitions. Insets: Film microstructure schematics (a) without and (b) with $\beta$-phase chain segments present.

In this report, we primarily consider the low temperature measurements $(T=15 \mathrm{~K})$ since the TA spectral features are more clearly resolved (room temperature spectra are compared in SI Figure S2). For the glassy-phase samples at $\Delta t=300 \mathrm{fs}$, a broad excited state band with a maximum at $\sim 1.60 \mathrm{eV}$ is observed and assigned to the $S_{1} \rightarrow S_{n}$ singlet transition. A relatively narrow peak subsequently emerges around $2.10 \mathrm{eV}$, superimposed on the $S_{1} \rightarrow S_{n}$ band and 
resolved within the first 500 fs following excitation. This peak is assigned to polaron absorption, in line with previous spectroelectrochemical studies of doped PFO and the closely related polyfluorene PF6. ${ }^{63,69}$ The observation demonstrates the ultrafast photogeneration of polarons in neat PFO, consistent with previous TA measurements, ${ }^{7,55,70}$ and also provides an explanation for why PFO is such a poor host material for optical gain when used in conjunction with the green emitter poly(9,9-dioctylfluorene-co-benzothiadiazole) ${ }^{71}$ A second narrow peak/shoulder at around $1.5 \mathrm{eV}$ becomes evident from $\Delta t \sim 1 \mathrm{ps}$, which is assigned to $T_{1} \rightarrow T_{n}$ triplet absorption, on the basis of its long lifetime, sensitivity to oxygen and the lack of any accompanying charge related infrared activated vibrations. ${ }^{48,53-55}$

For the samples containing $\beta$-phase chain segments, an additional set of TA peaks appear at $1.93 \mathrm{eV}$ and $1.42 \mathrm{eV}$, red-shifted by $\sim 0.2$ and $0.1 \mathrm{eV}$ respectively and narrowed compared to the glassy-phase polaron and triplet counterparts. The assignment of these peaks to polarons and triplets localised on $\beta$-phase chain segments follows from their lower energy and narrower linewidths, akin to the additional $\beta$-phase transitions in steady-state PFO absorption spectra. The $\beta$-phase polaron assignment has previously been confirmed by the expected PIAdetected magnetic resonance for a doublet. ${ }^{72}$ Tellingly, the $S_{1} \rightarrow S_{n}$ band is also broadened on the low energy edge due to the presence of a red-shifted $\beta$-phase singlet absorption contribution. The larger red-shift of the $\beta$-phase polaron vs triplet is consistent with greater localisation of the triplet state; a common feature of conjugated aromatic systems.

Taking a similar approach to that used for the steady-state absorption spectra, the various glassy- and $\beta$-phase absorption species that comprise the TA spectra were also isolated through fitting. Typical fitting profiles used for the excited state species are shown in the lower panels of Figure 2, with further details described in the experimental section. The ability to spectrally resolve signals from different excited state species, and thereby distinguish signals originating from glassy and $\beta$-phase chain segments, allows the impacts of 
conformation and microstructure on polaron photogeneration to be elucidated and quantified as presented further on.

Additional TA measurements, under selective excitation of $\beta$-phase chain segments at 437 $\mathrm{nm}$, were also performed on both the $8 \%$ and $23 \% \beta$-phase samples $(\beta$-phase fraction calculations are detailed in the SI) and are shown in Figure 3. Selective excitation, at $385 \mathrm{~nm}$ vs $437 \mathrm{~nm}$, provides a comparison of polaron photogeneration originating from majority glassy-phase excited states (and a minority of $\beta$-phase chain segments when present) vs $\beta$ phase only excited states respectively. Following excitation at $437 \mathrm{~nm}$, Figure 3 (b) and (c), the polaron and triplet contributions associated with the $\beta$-phase chain segments are more clearly resolved. 


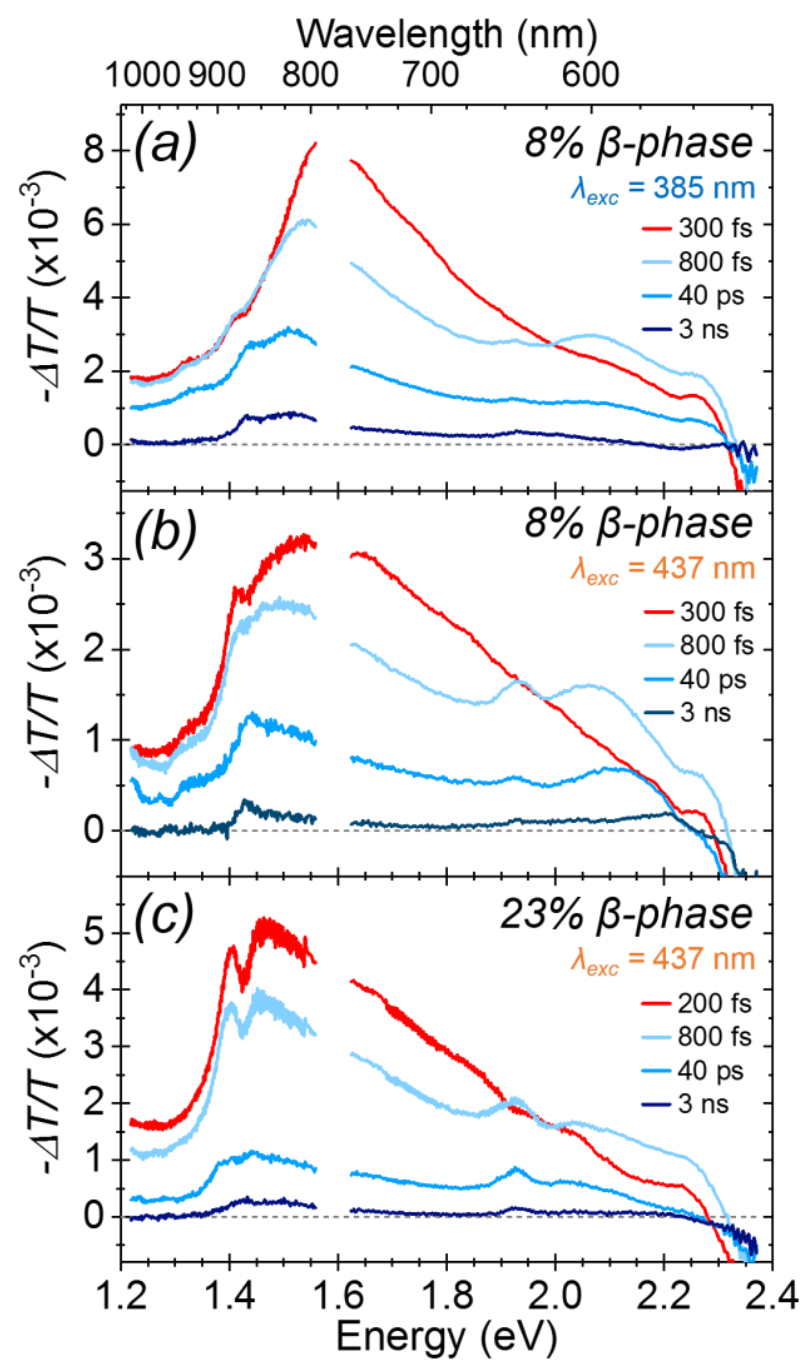

Figure 3. Low temperature $(T=15 \mathrm{~K})$ transient absorption spectra for $8( \pm 2) \% \beta$-phase PFO thin films excited at (a) $385 \mathrm{~nm}$ and (b) $437 \mathrm{~nm}$; and (c) 23 ( \pm 2) \% $\beta$-phase PFO excited at $437 \mathrm{~nm}$, for varying pump-probe delay times at a pump fluence of $30 \mu \mathrm{J} \mathrm{cm}^{-2}$.

TA spectra are presented for a pump fluence of $30 \mu \mathrm{J} \mathrm{cm}^{-2}$, where the signal intensityfluence response across the photoinduced absorption band is linear (shown in SI Figure S3). It is also noted from Figure S3 that the intensity-fluence response becomes sub-linear at higher fluence, with an inflection point at $\sim 100 \mu \mathrm{J} \mathrm{cm}^{-2}$, consistent with previous TA studies of PFO. ${ }^{7}$

In order to test the longevity of photogenerated polarons with varying microstructure, photoinduced absorption spectra of glassy-phase and $\beta$-phase PFO thin films were also 
obtained and are displayed in Figure 4. There is close correspondence between the $\Delta t=3 \mathrm{~ns}$ TA and PIA spectra, which are both consistent with previous PIA measurements of PFO films, ${ }^{53,54}$ confirming the identification of polaron and triplet peaks on ultrafast timescales.

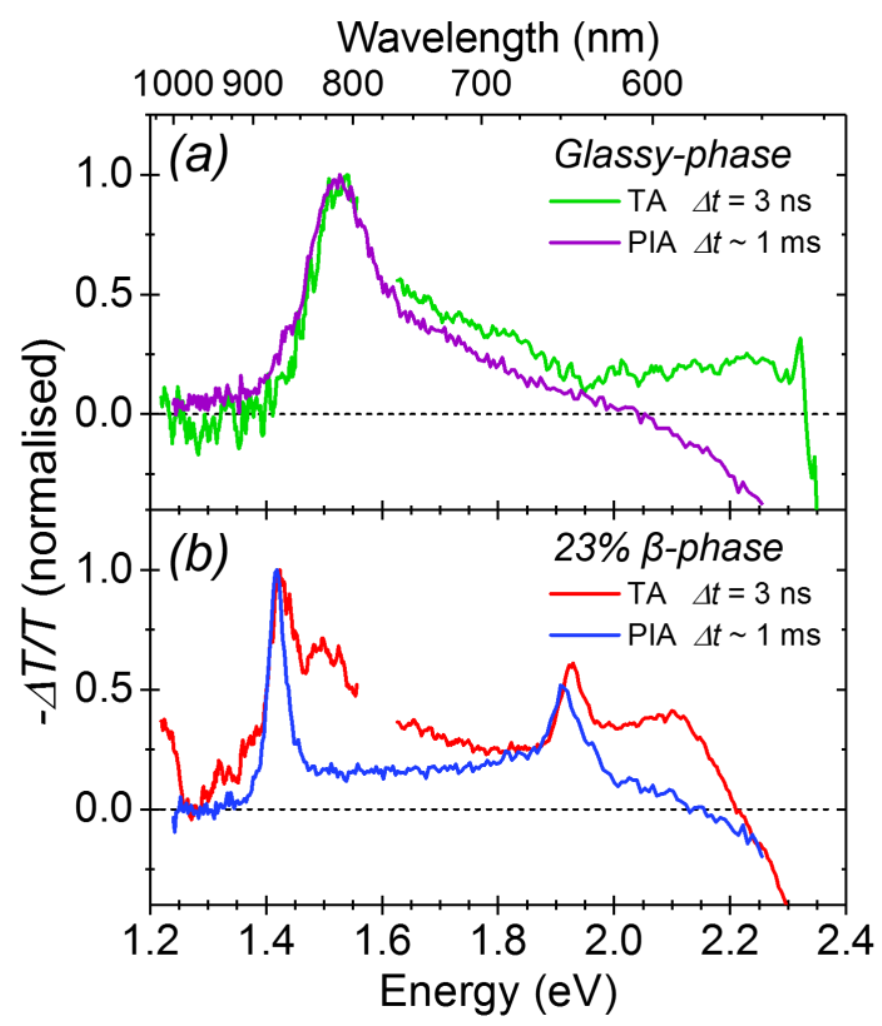

Figure 4. Low temperature ultrafast transient absorption (TA) and continuous-wave photoinduced absorption (PIA) spectra for (a) glassy-phase and (b) $23( \pm 2) \% \beta$-phase PFO thin films. TA spectra are shown for a pump-probe delay of $3 \mathrm{~ns}$ at $T=15 \mathrm{~K}(385 \mathrm{~nm}$ excitation, pump fluence $=30 \mu \mathrm{J}$ $\left.\mathrm{cm}^{-2}\right)$. PIA spectra were recorded at a modulation frequency of $170 \mathrm{~Hz}$, for $406 \mathrm{~nm}$ excitation at $\mathrm{T}=$ $12 \mathrm{~K}$.

Notably, for the $\beta$-phase PFO sample, the sharp $\beta$-phase polaron signal remains even for the long ( 1 ms) timescales probed by PIA. In contrast, no long-lived polarons are visible in the PIA of glassy-phase PFO. A similar observation is noted for the $\beta$-phase sample, where the glassy-phase polaron observed on the fs-ns timescales of TA measurements is also lost at the longer timescale of PIA. The glassy-phase PIA spectrum is instead dominated by the $T_{1} \rightarrow T_{n}$ triplet absorption. The variation in microstructure due to the presence of segments adopting 
the $\beta$-phase conformation has a significant impact upon both the initial population, evolution of said population and the subsequent lifetime of photogenerated polarons and triplets.

Here, there is further evidence that $\beta$-phase chain segments act as highly efficient energetic traps, in line with previous reports: ${ }^{32,49,73}$ TA spectra show a relative reduction in glassyphase vs $\beta$-phase polaron and triplet peaks over time, which indicate polaron and triplet migration from glassy-phase to $\beta$-phase sites for $\beta$-phase containing samples. Then, by the ms timescale of PIA measurements, these glassy-phase species are completely absent while $\beta$ phase species remain. Isolation of triplets and polarons on $\beta$-phase chain segments leads to reduced mobility and hence the lower rate of recombination and/or losses due to migration, explaining their longer lifetimes vs glassy-phase species. ${ }^{53}$ The $\beta$-phase polaron and triplet signals are also narrower than their glassy-phase counterparts, reflecting the reduced conformational variation within the rigid $\beta$-phase chain segments and the resulting narrower energetic density of states. The significantly narrower linewidth of the $\beta$-phase vs glassyphase polaron is also qualitatively consistent with the longer observed polaron lifetime, on the order of ms rather than ns. At higher energies, all PIA spectra show a positive $\Delta T / T$ signal, attributed to residual background steady-state PL.

Measured TA spectra are superpositions of overlapping singlet, polaron and triplet excited state absorptions. By fitting spectra and accounting for the different excited state absorption cross-sections, excited state populations as a function of pump-probe delay can be extracted. Further details are described in detail in the experimental section. Spectral fits and resulting excited state kinetics are presented in SI Figures S2 \& S4-S7.

A discussion of the TA spectral fitting methodology is provided in the supporting information. Excited state kinetics presented in SI Figures S5 and S6 reveal that the singlet 
population peaks at early times as expected at $\Delta t \sim 200-300 \mathrm{fs}$. Meanwhile polaron and triplet populations peak later at $500 \mathrm{fs}-1$ ps and 1-6 ps respectively (SI Figure S5-S7), depending on the sample. This observation confirms the different timescales of polaron and triplet formation. For the following decay in the polaron signal, there is some variation but no obvious trends with varying $\beta$-phase fraction and excitation wavelength (SI Figure S7). The excited state kinetics provide a quantitative yield for the generation of polarons and triplets from the initial photoexcited singlet population. Figure 5 shows the polaron generation yield for samples of varying $\beta$-phase fraction measured at low pump fluence, under the selective excitation of glassy- and $\beta$-phase chain segments. Remarkably, the polaron yield increases by $~ 3-4$ times in samples containing both glassy and $\beta$-phase $\left(\phi_{p o l}=\right.$ $4.4-6.3 \%)$ compared to pure glassy-phase samples $\left(\phi_{p o l}=1.6 \%\right)$. This is equally true in $\beta$ phase samples for both selective excitation of glassy-phase chains (and a minority of $\beta$-phase segments when present) at $385 \mathrm{~nm}$ and the lower-energy $\beta$-phase segments only at $437 \mathrm{~nm}$, with the $8 \% \beta$-phase sample showing a markedly higher polaron generation for excitation of $\beta$-phase chain segments alone. 


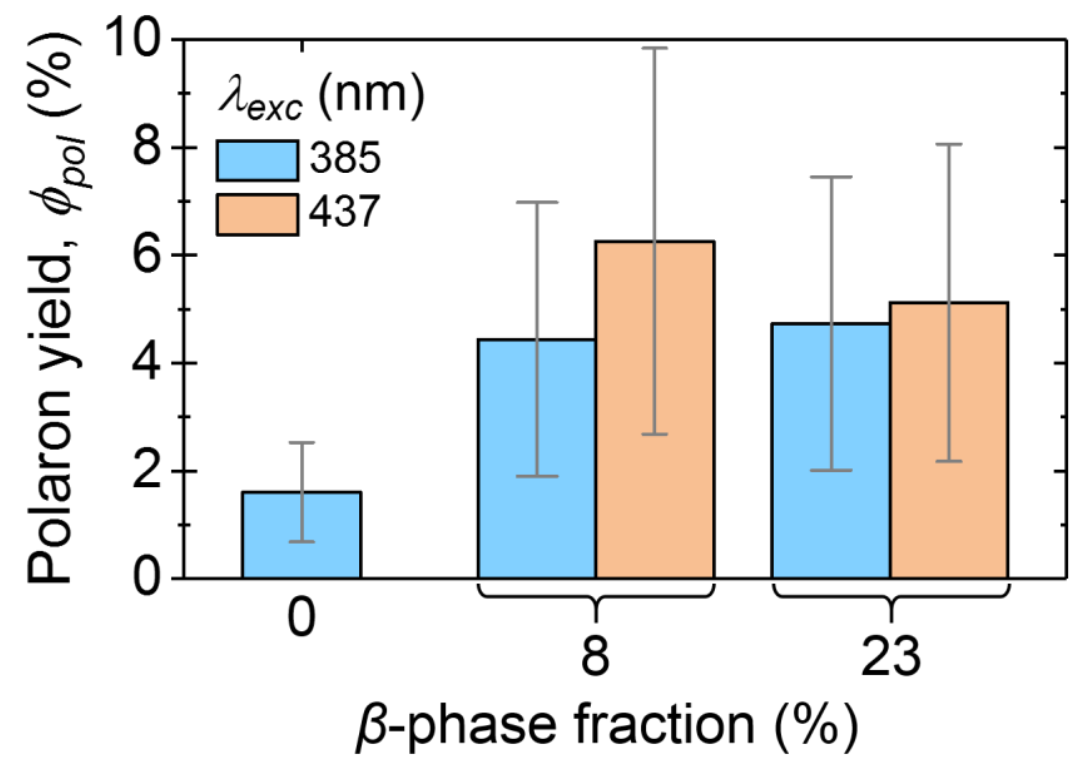

Figure 5. Photogenerated polaron yields for PFO thin films with varying $\beta$-phase fraction $(0,8 \pm 2 \&$ $23 \pm 2 \%)$. Yields are obtained from TA spectra $(T=15 \mathrm{~K})$, and compared for selective excitation of glassy-phase (and a minority of $\beta$-phase when present) chain segments at $\lambda_{\text {exc }}=385 \mathrm{~nm}$ (blue bars) and $\beta$-phase chain segments only at $\lambda_{\text {exc }}=437 \mathrm{~nm}$ (orange bars), for a pump fluence $=30 \mu \mathrm{J} \mathrm{cm}^{-2}$.

Table 2. Summary of photogenerated polaron and triplet yields (in \%) for PFO thin films with varying $\beta$-phase fraction, following excitation at $385 \mathrm{~nm}$ and $437 \mathrm{~nm}$ at varying temperature and pump fluence. Each yield value is quoted with a percentage uncertainty of $\pm 60 \%$.

\begin{tabular}{|c|c|c|c|c|c|c|}
\hline \multirow[b]{2}{*}{$T(K)$} & \multirow[b]{2}{*}{ 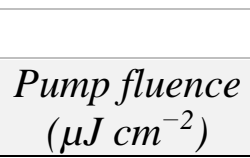 } & \multirow[b]{2}{*}{$\begin{array}{c}\beta \text {-phase } \\
\text { fraction (\%) }\end{array}$} & \multicolumn{2}{|c|}{ Polaron yield, $\phi_{p o l}(\%)$} & \multicolumn{2}{|c|}{ Triplet yield, $\phi_{\text {trip }}(\%)$} \\
\hline & & & $\begin{array}{c}\lambda_{e x c}=385 \\
n m\end{array}$ & $\begin{array}{c}\lambda_{e x c}=437 \\
n m\end{array}$ & $\begin{array}{c}\lambda_{e x c}=385 \\
n m\end{array}$ & $\begin{array}{c}\lambda_{e x c}=437 \\
n m\end{array}$ \\
\hline \multirow[t]{3}{*}{15} & \multirow[t]{3}{*}{30} & 0 & 1.6 & - & 6.5 & - \\
\hline & & $8( \pm 2)$ & 4.4 & 6.3 & 10 & 7.4 \\
\hline & & $23( \pm 2)$ & 4.7 & 5.1 & 9.7 & 4.2 \\
\hline \multirow[t]{3}{*}{15} & \multirow[t]{3}{*}{180} & 0 & 1.0 & - & 6.3 & - \\
\hline & & $8( \pm 2)$ & 3.0 & 8.2 & 7.3 & 4.6 \\
\hline & & $23( \pm 2)$ & 4.1 & 5.6 & 7.0 & 4.6 \\
\hline \multirow[t]{3}{*}{290} & \multirow[t]{3}{*}{$30-40$} & 0 & 1.5 & - & 3.3 & - \\
\hline & & $7( \pm 2)$ & - & 2.6 & - & 3.1 \\
\hline & & $23( \pm 2)$ & - & 7.0 & - & 1.8 \\
\hline \multirow[t]{3}{*}{290} & \multirow[t]{3}{*}{$180-230$} & 0 & 1.6 & - & 3.6 & - \\
\hline & & $7( \pm 2)$ & - & 3.0 & - & 3.1 \\
\hline & & $23( \pm 2)$ & - & 4.9 & - & 3.4 \\
\hline
\end{tabular}


Photogenerated yields of polarons and triplets from excited state singlets are summarised in Table 2, for a range of measurements taken at varying pump fluence and temperature. PFO polaron yield values are comparable to those previously reported for other neat polymers, on the order of $1-10 \% .^{3,5,8,12,13}$ Upon the introduction of $\beta$-phase into the thin film microstructure, a definite increase in polaron generation yield is evident and persistent across all measurement conditions. For low temperature measurements at higher pump fluence, where the signal-fluence dependence is sub-linear (SI Figure S3), a 3-8 times increase in polaron yield is again observed for samples containing $\beta$-phase. Similarly, for room temperature measurements, a 2-4 times increase in polaron yield is observed for $\beta$-phase samples at both low and high fluence. Curiously, no consistent trend is found for the increase in polaron yield vs the fraction of $\beta$-phase chain segments, with the $8 \%$ and $23 \% \beta$-phase samples giving similar results. Were an argument to be formed which related the increase in polaron yield to the number of glassy $/ \beta$-phase interfaces, this result would perhaps suggest that clustering of the $\beta$-phase chain segments may be occurring for larger $\beta$-phase concentrations. However, as discussed further on, other factors are also likely to play a role. What is clear is that across all measurements at low and room temperature and low and high fluence, $\beta$-phase-containing samples have higher polaron yields compared to glassy-phase samples by a factor of 3.4 on average: $\phi_{p o l}=4.9 \%$ vs $1.4 \%$, with standard deviations of 1.7 $\%$ and $0.3 \%$ obtained across 12 and 4 measurements respectively. The results confirm the influence of conformation on polaron generation in $\mathrm{PFO}$, and further support the view that a microstructure containing chains with ordered and disordered molecular conformations can lead to enhanced polaron generation.

Finally it is noted that samples containing $\beta$-phase segments consistently yield more 'secondary' species (polaron and triplets) in general. Interestingly, the balance between polaron and triplet generation is dependent upon the environment of the initial excitation, 
with the yield of polarons generally higher and triplet yield lower following $\beta$-phase excitation at $437 \mathrm{~nm}$ than glassy-phase at $385 \mathrm{~nm}$ (visualised in SI Figure S8).

\section{Discussion}

The key finding of this study is that PFO thin films containing a fraction of chain segments adopting the highly-ordered $\beta$-phase conformation in addition to twisted, disordered glassyphase conformations, generate significantly higher populations of polarons than glassy-phase only samples from photoexcitation. Notably, the increase in polaron generation is retained even when exciting the lower-energy $\beta$-phase chain segments only.

Selective excitation of PFO at $437 \mathrm{~nm}$ initially generates excitons exclusively on the $\beta$-phase chain segments (glassy-phase absorption is negligible at this wavelength). Yet, polaron signals from both $\beta$ - and glassy-phases are still clearly observed (SI Figure S4 (e) \& (f)), with polaron signals seen in $100 \%$ glassy-phase samples closely overlapping the signals seen in $\beta$ phase samples. Furthermore, both polaron peaks are first resolved on the same ultrafast timescale, showing that glassy and $\beta$-phase polarons form concurrently. Considered together this is compelling evidence that exciton dissociation occurs at the interfaces between glassy and $\beta$-phase chain segments. It follows that this interfacial exciton dissociation is the origin of the enhanced polaron generation in samples containing $\beta$-phase chain segments.

One interpretation for why polaron generation is enhanced at glassy/ $\beta$-phase interfaces is that the sharp energetic offsets between the two conformational species drives spatial separation of charges. The hypothesis is drawn from similar studies of the effect of microstructure on polaron generation in P3HT by both Paquin et al., ${ }^{12}$ and Reid et al., ${ }^{13}$ who proposed that the local energetic offsets between amorphous and crystalline regions were the driver for enhanced exciton dissociation in semicrystalline vs polycrystalline (where amorphous regions 
are absent) samples. As well as discussing further the effects of local energetic offsets, additional, not necessarily mutually exclusive, explanations for the increased exciton dissociation are considered. These are namely electron delocalisation and disparities between electron and hole charge carrier mobilities.

Firstly, several reports have found evidence to suggest that photoexcitations in conjugated polymers are initially highly spatially delocalised on ultrafast timescales. ${ }^{21,74,75}$ For such cases, significant spatial separation of the bound charges within excitons can lead to dissociation and polaron formation in neat polymer films. Theoretical studies of donor:acceptor systems have also suggested that introducing local energy fluctuations at D:A interfaces, akin to the large energetic offsets exhibited at glassy/ $\beta$-phase interfaces, increases the likelihood of coherence between exciton and charge-transfer/polaron states on similarly ultrafast timescales. ${ }^{24}$ This may be the case in PFO samples containing $\beta$-phase segments, while the higher polarizability and electronic delocalisation of the $\beta$-phase, in addition to the mismatch in conjugation lengths of the glassy and $\beta$-phases, ${ }^{48,63}$ may too act to enhance spatial separation of charges within excitons formed at or near to glassy/ $\beta$-phase interfaces. Again examining the effect of energetic offsets, the driving forces for photoinduced charge transfer, in the context of more localised excitations, can also be considered by examining molecular orbital energy levels. One of the most well-known properties of the $\beta$-phase in PFO is its ability to act as a highly efficient energetic trap, with efficient exciton and charge carrier transfer from glassy- to $\beta$-phase chain segments observed in photo- and electroluminescence measurements. ${ }^{32,49,73}$ These observations mean that glassy/ $\beta$-phase interfaces are thought to form type I heterojunctions that favour exciton migration to the $\beta$-phase over charge separation, with both $\beta$-phase HOMO and LUMO levels lying within those of the glassy-phase. Experimental determinations of the relative positions of the $\beta$-phase HOMO and LUMO levels are, however, inconclusive - spectroelectrochemical hole doping of $\beta$ - 
phase PFO samples has shown that the $\beta$-phase oxidation potential is lower than the glassyphase by $\Delta E \approx 100-150 \mathrm{meV},{ }^{63}$ indicative of a shallower HOMO level; and photocurrent and impedance spectroscopies suggest that the $\beta$-phase HOMO is also shallower by $\Delta E \approx 300$ meV. ${ }^{76}$ Meanwhile, trap-filling thermally stimulated current measurements exhibit no evidence of a difference in glassy and $\beta$-phase HOMO levels, but find the LUMO of the $\beta$ phase to be deeper than the glassy-phase by $\Delta E \approx 120 \mathrm{meV} .{ }^{32}$ Given this uncertainty, in combination with the local variation in HOMO and LUMO energies of glassy-phase chains due to conformational variation, it is reasonable to speculate that within a PFO film there may be a (small) fraction of glassy/ $\beta$-phase interfaces which form type II rather than type I heterojunctions, where offsets between HOMO or LUMO levels of neighbouring glassy and $\beta$-phase chain segments favour charge separation over energy transfer. If this were the case for only a small fraction of interfaces, it could explain the enhanced polaron yield in $\beta$-phase containing films.

In the case of semicrystalline P3HT, where type I heterojunctions are again expected between ordered and disordered regions, it has also been proposed that polaron generation is enhanced at ordered/disordered interfaces as a result of a mismatch in hole and electron mobilities, increasing the probability of spatial separation of charges rather than exciton migration. ${ }^{13} \mathrm{~A}$ similar argument may equally apply to PFO. However, there is much variation in the hole and electron mobilities of PFO quoted in the literature spanning several orders of magnitude and comparatively few reports of electron mobilities, ${ }^{32,77-81}$ thus making reliable assessment difficult. This noted, reports where both charge carrier mobilities were determined, suggest a large mismatch between hole and electron mobilities. ${ }^{80-82}$ Moreover, if there are differences in the mobilities of the same charge carrier type between glassy and $\beta$-phases, this may act to further increase the likelihood of spatial separation of charges at the glassy/ $\beta$-phase interface. 
Testing this speculation experimentally would be very challenging, due to the macroscopic nature of the mobility measurements and the difficulty in isolating $\beta$-phase segments.

It is noted that the assignment of polaron signals to holes or electrons is also not possible, as a high degree of overlap between hole and electron absorption signals is expected, as previously observed from electrochemical doping of the closely related polyfluorene PF6 (hexyl rather than octyl side-chains) ${ }^{69}$ As a result, it is not possible to know whether and to what extent the formation of a particular polaron within a particular conformation segment is favoured.

While various postulations can be made to explain why exciton dissociation is enhanced at glassy/ $\beta$-phase interfaces, their size and order of importance is as yet unknown. What is clear, and consistent with other system studies, is that the introduction of varying structural order, which provides a gradient in both the energetic landscape and degree of electronic delocalisation, is key to driving enhanced exciton generation in conjugated polymers.

\section{Conclusion}

In conclusion, the results presented in this study reinforce the key relationship between microstructure and exciton dissociation in conjugated polymers. Results of ultrafast transient absorption spectroscopy show that introducing small fractions of highly-ordered $\beta$-phase chain segments into otherwise disordered, glassy films of poly(9,9-dioctylfluorene) significantly increases the yield of polarons formed by ultrafast exciton dissociation of singlet excited states, on average by a factor of 3.4. Selective photoexcitation of the lower energy $\beta$ phase species only was found to produce as many polarons as when pumping a majority of the higher energy glassy-phase chain segments. Furthermore, dissociation of the $\beta$-phase excited state singlets produced higher energy glassy-phase polarons on the same femtosecond 
timescale as $\beta$-phase polarons. Based on these findings, the increase in polaron generation in $\beta$-phase containing films is attributed to exciton dissociation at interfaces between glassy and $\beta$-phase chain segments. Increased polaron generation at these interfaces is suspected to be driven by a combination of the large energetic offsets between glassy and $\beta$-phase chain segments, the higher electronic delocalisation of the $\beta$-phase, and asymmetry in charge carrier mobilities. Our observations and proposals are consistent with those previously reported for P3HT, where focus was made on increased charge separation at interfaces between amorphous and crystalline regions. In this regard, the relationship between energetic offsets, delocalisation and increased exciton dissociation appears to be widely applicable to other conjugated polymer systems. Consequently, the role of microstructural variation within neat polymers in separating charge should be considered in the design of future materials and applications where exciton dissociation is desired. Likewise, if polaron generation is an unwanted by-product of photoexcitation, heterogeneous microstructures within neat conjugated polymers containing multiple species with large energetic differences and variations in delocalisation should be avoided.

\section{Acknowledgements}

NJC, JN, DDCB and PNS acknowledge funding by the UK Engineering and Physical Sciences Research Council (EPSRC) for the Plastic Electronics Materials Doctoral Training Centre (EP/G037515/1). The authors also gratefully acknowledge the Science and Technology Facilities Council (STFC) for providing beam time on the ULTRA setup at the Central Laser Facility in the Research Complex at Harwell. The authors declare no competing financial interest. 


\section{Supporting Information Description}

Steady-state absorption fitting and $\beta$-phase fraction calculation details, additional TA spectra at room and low temperature (with spectral fitting), TA kinetics of excited state species, fluence dependence of TA signals, additional polaron and triplet yield visualisations, and discussion of TA spectral fitting methodology

\section{Author Description}

Corresponding Author:

*E-mail: (N.J.C.) n.cheetham13@imperial.ac.uk.

*E-mail: (P.N.S.) paul.stavrinou@ @incoln.ox.ac.uk.

*E-mail: (S.C.H.) shayes@ucy.ac.cy.

ORCID:

Nathan Cheetham: 0000-0002-2259-1556

Paul Stavrinou: 0000-0001-6075-2587

Sophia Hayes: 0000-0003-0238-6915

Aleksandr Perevedentsev: 0000-0003-0146-3560

Laura-Isabelle Dion-Bertrand: 0000-0001-7435-4002

Michael Towrie: 0000-0002-1305-701X

Anthony Parker: 0000-0003-3094-9762

Jenny Nelson: 0000-0003-1048-1330

Donal Bradley: 0000-0001-8713-5060 


\section{References}

1. Marks, R. N.; Halls, J. J. M.; Bradley, D. D. C.; Friend, R. H.; Holmes, A. B. The Photovoltaic Response in Poly(p-Phenylene Vinylene) Thin-Film Devices. J. Phys. Condens. Matter 1994, 6, 13791394.

2. Alvarado, S. F.; Seidler, P. F.; Lidzey, D. G.; Bradley, D. D. C. Direct Determination of the Exciton Binding Energy of Conjugated Polymers Using a Scanning Tunneling Microscope. Phys. Rev. Lett. 1998, $81,1082-1085$.

3. Tautz, R.; Da Como, E.; Limmer, T.; Feldmann, J.; Egelhaaf, H.-J.; von Hauff, E.; Lemaur, V.; Beljonne, D.; Yilmaz, S.; Dumsch, I.; Allard, S.; Scherf, U. Structural Correlations in the Generation of Polaron Pairs in Low-Bandgap Polymers for Photovoltaics. Nat. Commun. 2012, 3, 970.

4. Clarke, T. M.; Durrant, J. R. Charge Photogeneration in Organic Solar Cells. Chem. Rev. 2010, 110, $6736-67$.

5. Reid, O. G.; Rumbles, G. Quantitative Transient Absorption Measurements of Polaron Yield. J. Phys. Chem. Lett. 2013, 4, 2348-2355.

6. Reid, O. G.; Pensack, R. D.; Song, Y.; Scholes, G. D.; Rumbles, G. Charge Photogeneration in Neat Conjugated Polymers. Chem. Mater. 2013, 26, 561-575.

7. Stevens, M.; Silva, C.; Russell, D.; Friend, R. Exciton Dissociation Mechanisms in the Polymeric Semiconductors Poly(9,9-Dioctylfluorene) and Poly(9,9-Dioctylfluorene-Co-Benzothiadiazole). Phys. Rev. B 2001, 63, 165213.

8. Silva, C.; Dhoot, A.; Russell, D.; Stevens, M.; Arias, A.; MacKenzie, J.; Greenham, N.; Friend, R.; Setayesh, S.; Müllen, K. Efficient Exciton Dissociation via Two-Step Photoexcitation in Polymeric Semiconductors. Phys. Rev. B 2001, 64, 125211.

9. Silva, C.; Russell, D. M.; Dhoot, A. S.; Herz, L. M.; Daniel, C.; Greenham, N. C.; Arias, A. C.; Setayesh, S.; Müllen, K.; Friend, R. H. Exciton and Polaron Dynamics in a Step-Ladder Polymeric Semiconductor: The Influence of Interchain Order. J. Phys. Condens. Matter 2002, 14, 9803-9824.

10. Kraabel, B.; Klimov, V. I.; Kohlman, R.; Xu, S.; Wang, H.; Mcbranch, D. W. Unified Picture of the Photoexcitations in Phenylene-Based Conjugated Polymers : Universal Spectral and Dynamical Features 
in Subpicosecond Transient Absorption. Phys. Rev. B 2000, 61, 8501-8515.

11. De Sio, A.; Troiani, F.; Maiuri, M.; Réhault, J.; Sommer, E.; Lim, J.; Huelga, S. F.; Plenio, M. B.; Rozzi, C. A.; Cerullo, G.; Molinari, E.; Lienau, C. Tracking the Coherent Generation of Polaron Pairs in Conjugated Polymers. Nat. Commun. 2016, 7, 13742.

12. Paquin, F.; Latini, G.; Sakowicz, M.; Karsenti, P. L.; Wang, L.; Beljonne, D.; Stingelin, N.; Silva, C. Charge Separation in Semicrystalline Polymeric Semiconductors by Photoexcitation: Is the Mechanism Intrinsic or Extrinsic? Phys. Rev. Lett. 2011, 106, 1-4.

13. Reid, O. G.; Malik, J. A. N.; Latini, G.; Dayal, S.; Kopidakis, N.; Silva, C.; Stingelin, N.; Rumbles, G. The Influence of Solid-State Microstructure on the Origin and Yield of Long-Lived Photogenerated Charge in Neat Semiconducting Polymers. J. Polym. Sci. Part B Polym. Phys. 2012, 50, $27-37$.

14. Paquin, F.; Rivnay, J.; Salleo, A.; Stingelin, N.; Silva, C. Multi-Phase Semicrystalline Microstructures Drive Exciton Dissociation in Neat Plastic Semiconductors. J. Mater. Chem. C 2015, 3, 10715-10722.

15. Di Nuzzo, D.; Viola, D.; Fischer, F. S. U.; Cerullo, G.; Ludwigs, S.; Da Como, E. Enhanced Photogeneration of Polaron Pairs in Neat Semicrystalline Donor-Acceptor Copolymer Films via Direct Excitation of Interchain Aggregates. J. Phys. Chem. Lett. 2015, 6, 1196-1203.

16. Herrmann, D.; Niesar, S.; Scharsich, C.; Köhler, A.; Stutzmann, M.; Riedle, E. Role of Structural Order and Excess Energy on Ultrafast Free Charge Generation in Hybrid Polythiophene/Si Photovoltaics Probed in Real Time by Near-Infrared Broadband Transient Absorption. J. Am. Chem. Soc. 2011, 133, $18220-18233$.

17. Jones, M. L.; Huang, D. M.; Chakrabarti, B.; Groves, C. Relating Molecular Morphology to Charge Mobility in Semicrystalline Conjugated Polymers. J. Phys. Chem. C 2016, 120, 4240-4250.

18. Dixon, A. G.; Visvanathan, R.; Clark, N. A.; Stingelin, N.; Kopidakis, N.; Shaheen, S. E. Molecular Weight Dependence of Carrier Mobility and Recombination Rate in Neat P3HT Films. J. Polym. Sci. Part B Polym. Phys. 2017, 56, 31-35.

19. Noriega, R.; Rivnay, J.; Vandewal, K.; Koch, F. P. V; Stingelin, N.; Smith, P.; Toney, M. F.; Salleo, A. A General Relationship between Disorder, Aggregation and Charge Transport in Conjugated Polymers. Nat. Mater. 2013, 12, 1038-44. 
20. Kline, R. J.; McGehee, M. D.; Kadnikova, E. N.; Liu, J.; Fréchet, J. M. J.; Toney, M. F. Dependence of Regioregular Poly(3-Hexylthiophene) Film Morphology and Field-Effect Mobility on Molecular Weight. Macromolecules 2005, 38, 3312-3319.

21. Banerji, N. Sub-Picosecond Delocalization in the Excited State of Conjugated Homopolymers and Donor-acceptor Copolymers. J. Mater. Chem. C 2013, 1, 3052.

22. Pace, N. A.; Reid, O. G.; Rumbles, G. Delocalization Drives Free Charge Generation in Conjugated Polymer Films. ACS Energy Lett. 2018, 3, 735-741.

23. Park, K. H.; Son, S. Y.; Kim, J. O.; Kang, G.; Park, T.; Kim, D. Role of Disorder in the Extent of Interchain Delocalization and Polaron Generation in Polythiophene Crystalline Domains. J. Phys. Chem. Lett. 2018, 9, 3173-3180.

24. Bittner, E. R.; Silva, C. Noise-Induced Quantum Coherence Drives Photo-Carrier Generation Dynamics at Polymeric Semiconductor Heterojunctions. Nat. Commun. 2014, 5, 3119.

25. D’Avino, G.; Mothy, S.; Muccioli, L.; Zannoni, C.; Wang, L.; Cornil, J.; Beljonne, D.; Castet, F. Energetics of Electron-Hole Separation at P3HT/PCBM Heterojunctions. J. Phys. Chem. C 2013, 117, 12981-12990.

26. Hood, S. N.; Kassal, I. Entropy and Disorder Enable Charge Separation in Organic Solar Cells. J. Phys. Chem. Lett. 2016, 7, 4495.

27. Shi, L.; Lee, C. K.; Willard, A. P. The Enhancement of Interfacial Exciton Dissociation by Energetic Disorder Is a Nonequilibrium Effect. ACS Cent. Sci. 2017, 3, 1262-1270.

28. Grell, M.; Bradley, D. D. C.; Long, X.; Chamberlain, T.; Inbasekaran, M.; Woo, E. P.; Soliman, M. Chain Geometry, Solution Aggregation and Enhanced Dichroism in the Liquidcrystalline Conjugated Polymer Poly(9,9-Dioctylfluorene). Acta Polym. 1998, 49, 439-444.

29. Hill, J.; Heriot, S.; Worsfold, O.; Richardson, T.; Fox, A.; Bradley, D. D. C. Controlled Förster Energy Transfer in Emissive Polymer Langmuir-Blodgett Structures. Phys. Rev. B 2004, 69, 041303.

30. Grell, M.; Bradley, D. D. C.; Ungar, G.; Hill, J.; Whitehead, K. S. Interplay of Physical Structure and Photophysics for a Liquid Crystalline Polyfluorene. Macromolecules 1999, 32, 5810-5817.

31. Peet, J.; Brocker, E.; Xu, Y.; Bazan, G. C. Controlled $\beta$-Phase Formation in Poly(9,9-Di-n- 
Octylfluorene) by Processing with Alkyl Additives. Adv. Mater. 2008, 20, 1882-1885.

32. Lu, H. H.; Liu, C. Y.; Chang, C. H.; Chen, S. A. Self-Dopant Formation in Poly(9,9-Di-n-

Octylfluorene) via a Dipping Method for Efficient and Stable Pure-Blue Electroluminescence. Adv.

Mater. 2007, 19, 2574-2579.

33. Perevedentsev, A.; Chander, N.; Kim, J.-S.; Bradley, D. D. C. Spectroscopic Properties of Poly(9,9Dioctylfluorene) Thin Films Possessing Varied Fractions of $\beta$-Phase Chain Segments: Enhanced Photoluminescence Efficiency via Conformation Structuring. J. Polym. Sci., Part B 2016, 54, 19952006.

34. Zhang, Q.; Chi, L.; Hai, G.; Fang, Y.; Li, X.; Xia, R. An Easy Approach to Control $\beta$-Phase Formation in PFO Films for Optimized Emission Properties. Molecules 2017, 22, 315.

35. Bright, D. W.; Dias, F. B.; Galbrecht, F.; Scherf, U.; Monkman, A. P. The Influence of Alkyl-Chain Length on Beta-Phase Formation in Polyfluorenes. Adv. Funct. Mater. 2009, 19, 67-73.

36. Bright, D. W.; Galbrecht, F.; Scherf, U.; Monkman, A. P. $\beta$ Phase Formation in Poly(9,9-Di-nDecylfluorene) Thin Films. Macromolecules 2010, 43, 7860-7863.

37. Knaapila, M.; Bright, D. W.; Nehls, B. S.; Garamus, V. M.; Schweins, R.; Scherf, U.; Monkman, A. P. Development of Intermolecular Structure and Beta-Phase of Random Poly[9,9-Bis(2Ethylhexyl)Fluorene]-Co-(9,9-Dioctylfluorene) in Methylcyclohexane. Macromolecules 2011, 44, 6453-6460.

38. Zhao, S.; Liang, J.; Guo, T.; Wang, Y.; Chen, X.; Fu, D.; Xiong, J.; Ying, L.; Yang, W.; Peng, J.; Cao, Y. Formation of Poly(9,9-Dioctylfluorene) $\beta$-Phase by Incorporating Aromatic Moiety in Side Chain. Org. Electron. 2016, 38, 130-138.

39. Lin, J.-Y.; Zhu, W.-S.; Liu, F.; Xie, L.-H.; Zhang, L.; Xia, R.; Xing, G.-C.; Huang, W. A Rational Molecular Design of B-Phase Polydiarylfluorenes: Synthesis, Morphology, and Organic Lasers. Macromolecules 2014, 47, 1001-1007.

40. Liu, B.; Lin, J.; Liu, F.; Yu, M.; Zhang, X.; Xia, R.; Yang, T.; Fang, Y.; Xie, L.; Huang, W. A Highly Crystalline and Wide-Bandgap Polydiarylfluorene with B-Phase Conformation toward Stable Electroluminescence and Dual Amplified Spontaneous Emission. ACS Appl. Mater. Interfaces 2016, 8, 
21648-21655.

41. Liu, B.; Lin, J.; Yu, M.; Li, B.; Xie, L.; Ou, C.; Liu, F.; Li, T.; Lu, D.; Huang, W. Hereditary Character of Alkyl-Chain Length Effect on $\beta$-Phase Conformation from Polydialkylfluorenes to Bulky Polydiarylfluorenes. J. Phys. Chem. C 2017, 121, 19087-19096.

42. Hamilton, I.; Chander, N.; Cheetham, N. J.; Suh, M.; Dyson, M.; Wang, X.; Stavrinou, P. N.; Cass, M.; Bradley, D. D. C.; Kim, J. S. Controlling Molecular Conformation for Highly Efficient and Stable Deep-Blue Copolymer Light-Emitting Diodes. ACS Appl. Mater. Interfaces 2018, 10, 11070-11082.

43. Meazzini, I.; Behrendt, J. M.; Turner, M. L.; Evans, R. C. Targeted $\beta$-Phase Formation in Poly(Fluorene)-Ureasil Grafted Organic-Inorganic Hybrids. Macromolecules 2017, 50, 4235-4243.

44. Bai, L.; Liu, B.; Han, Y.; Yu, M.; Wang, J.; Zhang, X.; Ou, C.; Lin, J.; Zhu, W.; Xie, L.; Yin, C.; Zhao, J.; Wang, J.; Bradley, D. D. C.; Huang, W. Steric-Hindrance-Functionalized Polydiarylfluorenes: Conformational Behavior, Stabilized Blue Electroluminescence, and Efficient Amplified Spontaneous Emission. ACS Appl. Mater. Interfaces 2017, 9, 37856-37863.

45. Chunwaschirasiri, W.; Tanto, B.; Huber, D. L.; Winokur, M. J. Chain Conformations and Photoluminescence of Poly(Di-n-Octylfluorene). Phys. Rev. Lett. 2005, 94, 107402.

46. Bradley, D. D. C.; Grell, M.; Long, X.; Mellor, H.; Grice, A. W.; Inbasekaran, M.; Woo, E. P. Influence of Aggregation on the Optical Properties of a Polyfluorene. Proc. SPIE 1997, 3145, 254-259.

47. Stavrinou, P. N.; Ryu, G.; Campoy-Quiles, M.; Bradley, D. D. C. The Change in Refractive Index of Poly(9,9-Dioctylfluorene) Due to the Adoption of the $\beta$-Phase Chain Conformation. J. Phys. Condens. Matter 2007, 19, 466107.

48. Cadby, A. J.; Lane, P. A.; Mellor, H.; Martin, S. J.; Grell, M.; Giebeler, C.; Bradley, D. D. C. Film Morphology and Photophysics of Polyfluorene. Phys. Rev. B 2000, 62, 15604-15609.

49. Ariu, M.; Sims, M.; Rahn, M.; Hill, J.; Fox, a.; Lidzey, D.; Oda, M.; Cabanillas-Gonzalez, J.; Bradley, D. Exciton Migration in $\beta$-Phase Poly(9,9-Dioctylfluorene). Phys. Rev. B 2003, 67, 195333.

50. Shaw, P. E.; Ruseckas, A.; Peet, J.; Bazan, G. C.; Samuel, I. D. W. Exciton-Exciton Annihilation in Mixed-Phase Polyfluorene Films. Adv. Funct. Mater. 2010, 20, 155-161.

51. Panzer, F.; Bässler, H.; Köhler, A. Temperature Induced Order-Disorder Transition in Solutions of 
Conjugated Polymers Probed by Optical Spectroscopy. J. Phys. Chem. Lett. 2017, 8, 114-125.

52. Le Roux, F.; Bradley, D. D. C. Conformational Control of Exciton-Polariton Physics in Metal-Poly(9,9Dioctylfluorene)-Metal Cavities. Phys. Rev. B 2018, 98, 195306.

53. Ariu, M.; Lidzey, D. G.; Sims, M.; Cadby, A. J.; Lane, P. A.; Bradley, D. D. C. The Effect of Morphology on the Temperature-Dependent Photoluminescence Quantum Efficiency of the Conjugated Polymer Poly(9, 9-Dioctylfluorene). J. Phys. Condens. Matter 2002, 14, 9975-9986.

54. Hayer, A.; Khan, A.; Friend, R.; Köhler, A. Morphology Dependence of the Triplet Excited State Formation and Absorption in Polyfluorene. Phys. Rev. B 2005, 71, 241302.

55. Tamai, Y.; Ohkita, H.; Benten, H.; Ito, S. Singlet Fission in Poly (9,9'-Di-n-Octyl Fluorene) Films. J. Phys. Chem. C 2013, 117, 10277-10284.

56. Greetham, G. M.; Burgos, P.; Cao, Q.; Clark, I. P.; Codd, P. S.; Farrow, R. C.; George, M. W.; Kogimtzis, M.; Matousek, P.; Parker, A. W.; Pollard, M. R.; Robinson, D. A.; Xin, Z.; Towrie, M. ULTRA: A Unique Instrument for Time-Resolved Spectroscopy. Appl. Spectrosc. 2010, 64, 1311-1452.

57. Sims, M.; Bradley, D. D. C.; Ariu, M.; Koeberg, M.; Asimakis, A.; Grell, M.; Lidzey, D. G. Understanding the Origin of the $535 \mathrm{Nm}$ Emission Band in Oxidized Poly(9,9-Dioctylfluorene): The Essential Role of Inter-Chain/Inter-Segment Interactions. Adv. Funct. Mater. 2004, 14, 765-781.

58. Zhao, W.; Cao, T.; White, J. M. On the Origin of Green Emission in Polyfluorene Polymers: The Roles of Thermal Oxidation Degradation and Crosslinking. Adv. Funct. Mater. 2004, 14, 783-790.

59. Bouguer, P. Essai d'optique sur la gradation de la lumière. (Claude Jombert, 1729).

60. Lambert, J. H. Photometria sive de mensura et gradibus luminis, colorum et umbrae. (Eberhardt Klett, $1760)$.

61. Beer, A. Bestimmung Der Absorption Des Rothen Lichts in Farbigen Flüssigkeiten. Ann. Phys. 1852, $162,78-88$.

62. Barker, A. J.; Hodgkiss, J. M. Quantitative Decoupling of Excited-State Absorption Cross Section and Population via Pump-Probe Spectroscopy with a Strong Probe. Phys. Rev. Appl. 2015, 4, 024017.

63. Montilla, F.; Ruseckas, A.; Samuel, I. D. W. Absorption Cross-Sections of Hole Polarons in Glassy and 
ß-Phase Polyfluorene. Chem. Phys. Lett. 2013, 585, 133-137.

64. Montilla, F.; Ruseckas, A.; Samuel, I. D. W. Exciton-Polaron Interactions in Polyfluorene Films with $\beta$ Phase. J. Phys. Chem. C 2018, 122, 9766-9772.

65. Lee, C. L.; Yang, X.; Greenham, N. C. Determination of the Triplet Excited-State Absorption Cross Section in a Polyfluorene by Energy Transfer from a Phosphorescent Metal Complex. Phys. Rev. B 2007, 76, 245201.

66. Kallel, H.; Latini, G.; Paquin, F.; Rinfret, R.; Stingelin, N.; Silva, C. Background-Free Quasi-SteadyState Photoinduced Absorption Spectroscopy by Dual Optical Modulation. 2010, arXiv:1007.3035v2 [cond-mtrl-sci]. arXiv prepr. https://arxiv.org/abs/1007.3035 (accessed 05/19/2019) .

67. Perevedentsev, A.; Sonnefraud, Y.; Belton, C. R.; Sharma, S.; Cass, A. E. G.; Maier, S. a; Kim, J.; Stavrinou, P. N.; Bradley, D. D. C. Dip-Pen Patterning of Poly(9,9-Dioctylfluorene) ChainConformation-Based Nano-Photonic Elements. Nat. Commun. 2015, 6, 5977.

68. Huang, L.; Huang, X.; Sun, G.; Gu, C.; Lu, D.; Ma, Y. Study of $\beta$ Phase and Chains Aggregation Degrees in Poly(9,9-Dioctylfluorene) (PFO) Solution. J. Phys. Chem. C 2012, 116, 7993-7999.

69. Takeda, N.; Asaoka, S.; Miller, J. R. Nature and Energies of Electrons and Holes in a Conjugated Polymer, Polyfluorene. J. Am. Chem. Soc. 2006, 128, 16073-16082.

70. Virgili, T.; Marinotto, D.; Lanzani, G.; Bradley, D. D. C. Ultrafast Resonant Optical Switching in Isolated Polyfluorenes Chains. Appl. Phys. Lett. 2005, 86, 091113.

71. Zhang, Q.; Liu, J.; Wei, Q.; Guo, X.; Xu, Y.; Xia, R.; Xie, L.; Qian, Y.; Sun, C.; Lüer, L.; CabanillasGonzalez, J.; Bradley, D. D. C.; Huang, W. Host Exciton Confinement for Enhanced Förster-TransferBlend Gain Media Yielding Highly Efficient Yellow-Green Lasers. Adv. Energy Mater. 2018, 28, 1705824.

72. Cadby, A. J.; Lane, P. A.; Wohlgenannt, M.; An, C.; Vardeny, Z. V; Bradley, D. D. C. Optical Studies of Photoexcitations of Poly(9,9-Dioctyl Fluorene). Synth. Met. 2000, 111-112, 515-518.

73. Khan, A. L. T.; Sreearunothai, P.; Herz, L. M.; Banach, M. J.; Köhler, A. Morphology-Dependent Energy Transfer within Polyfluorene Thin Films. Phys. Rev. B 2004, 69, 085201.

74. Ruseckas, A.; Wood, P.; Samuel, I. D. W.; Webster, G. R.; Mitchell, W. J.; Burn, P. L.; Sundström, V. 
Ultrafast Depolarization of the Fluorescence in a Conjugated Polymer. Phys. Rev. B 2005, 72, 115214.

75. Banerji, N.; Cowan, S.; Vauthey, E.; Heeger, A. J. Ultrafast Relaxation of the Poly(3-Hexylthiophene) Emission Spectrum. J. Phys. Chem. C 2011, 115, 9726-9739.

76. Shi, X.; Nádaždy, V.; Perevedentsev, A.; Frost, J. M.; Wang, X.; von Hauff, E.; MacKenzie, R. C. I.; Nelson, J. Relating Chain Conformation to the Density of States and Charge Transport in Conjugated Polymers: The Role of the $\beta$-Phase in Poly(9,9-Dioctylfluorene). Phys. Rev. X 2019, 9, 021038.

77. Redecker, M.; Bradley, D. D. C.; Inbasekaran, M.; Woo, E. P. Nondispersive Hole Transport in an Electroluminescent Polyfluorene. Appl. Phys. Lett. 1998, 73, 1565.

78. Redecker, M.; Bradley, D. D. C.; Inbasekaran, M.; Woo, E. P. Mobility Enhancement through Homogeneous Nematic Alignment of a Liquid-Crystalline Polyfluorene. Appl. Phys. Lett. 1999, 74, 1400.

79. Foster, S. On the Influence of Physical and Chemical Structure on Charge Transport in Disordered Semiconducting Materials and Devices. (Imperial College London, 2013).

80. Pinner, D. J.; Friend, R. H.; Tessler, N. Transient Electroluminescence of Polymer Light Emitting Diodes Using Electrical Pulses. J. Appl. Phys. 1999, 86, 5116.

81. Liang, J.; Yu, L.; Zhao, S.; Ying, L.; Liu, F.; Yang, W.; Peng, J.; Cao, Y. Improving Efficiency and Color Purity of Poly(9,9-Dioctylfluorene) through Addition of a High Boiling-Point Solvent of 1Chloronaphthalene. Nanotechnology 2016, 27, 284001.

82. Chua, L.; Zaumseil, J.; Chang, J.-F.; Ou, E. C.-W.; Ho, P. K.-H.; Sirringhaus, H.; Friend, R. H. General Observation of N-Type Field-Effect Behaviour in Organic Semiconductors. Nature 2005, 434, 194-199. 
Table of contents graphic

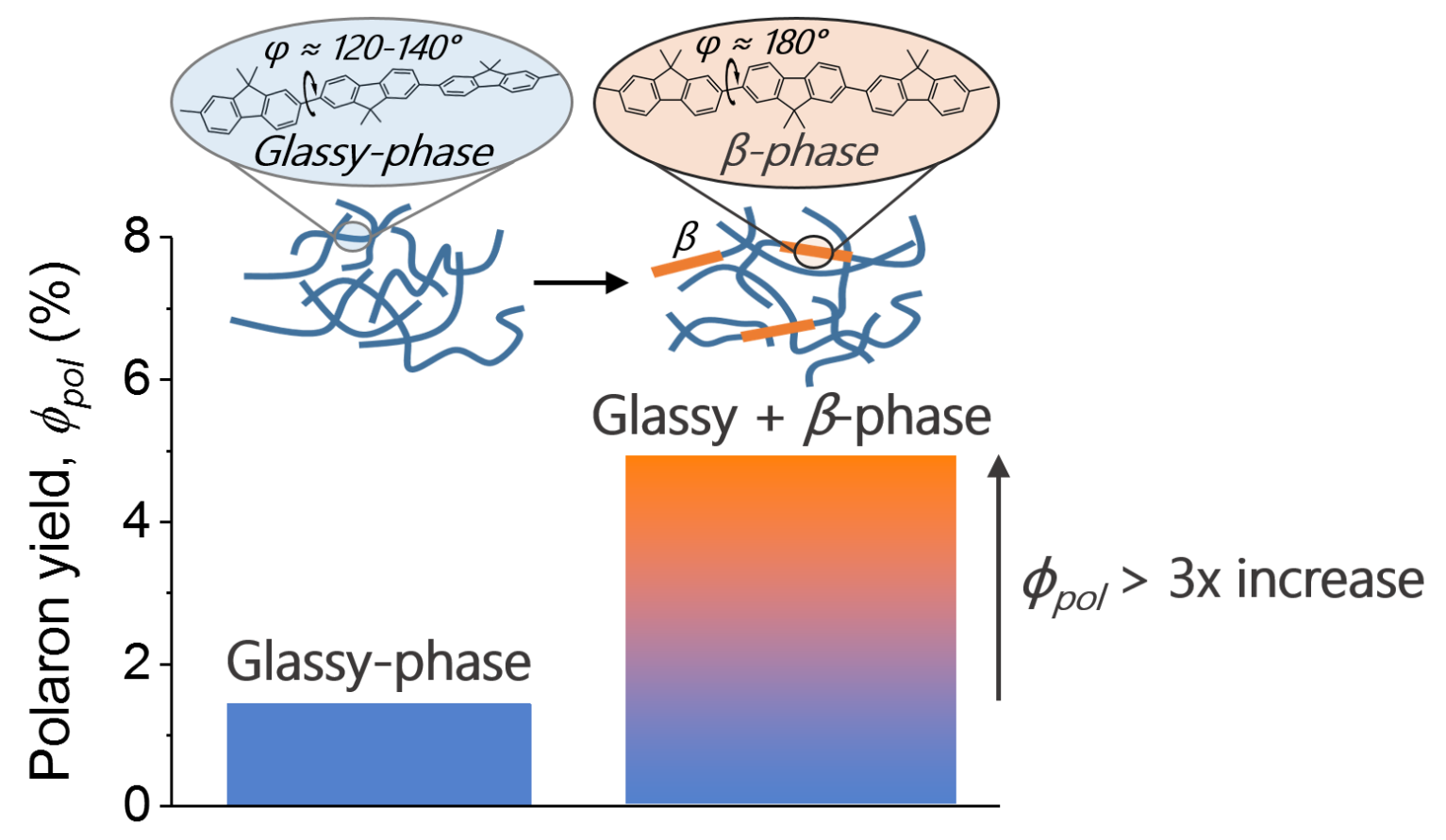

